\title{
Electron transport in a double quantum ring: Evidence of an AND gate
}

\author{
Santanu K. Maiti ${ }^{1,2, *}$ \\ ${ }^{1}$ Theoretical Condensed Matter Physics Division, Saha Institute of Nuclear Physics, \\ 1/AF, Bidhannagar, Kolkata-700 064, India \\ ${ }^{2}$ Department of Physics, Narasinha Dutt College, 129, Belilious Road, Howrah-711 101, India
}

\begin{abstract}
We explore AND gate response in a double quantum ring where each ring is threaded by a magnetic flux $\phi$. The double quantum ring is attached symmetrically to two semi-infinite one-dimensional metallic electrodes and two gate voltages, namely, $V_{a}$ and $V_{b}$, are applied, respectively, in the lower arms of the two rings which are treated as two inputs of the AND gate. The system is described in the tight-binding framework and the calculations are done using the Green's function formalism. Here we numerically compute the conductance-energy and current-voltage characteristics as functions of the ring-to-electrode coupling strengths, magnetic flux and gate voltages. Our study suggests that, for a typical value of the magnetic flux $\phi=\phi_{0} / 2\left(\phi_{0}=c h / e\right.$, the elementary flux-quantum) a high output current (1) (in the logical sense) appears only if both the two inputs to the gate are high (1), while if neither or only one input to the gate is high (1), a low output current (0) results. It clearly demonstrates the AND gate behavior and this aspect may be utilized in designing an electronic logic gate.
\end{abstract}

PACS No.: 73.23.-b; 73.63.Rt.

Keywords: Double quantum ring; Conductance; $I-V$ characteristic; AND gate.

*Corresponding Author: Santanu K. Maiti

Electronic mail: santanu.maiti@saha.ac.in 


\section{Introduction}

In the present age of nanoscience and technology, the study of electron transport through quantum confined systems has attracted a great deal of interest, both for application and research, in the field of developing nanoelectronics as well as spintronics. With the aid of present technological progress, simple looking quantum confined model systems like, quantum rings, quantum dots, arrays of quantum dots, etc, can be used extensively in designing nano devices, and, they are treated as the fundamental building blocks for future generation of nanoelectronics. The key idea of designing nanodevices is based on the concept of quantum interference effect, and it is generally preserved throughout the sample only for much smaller sizes, while the effect disappears for larger systems. A normal metal mesoscopic ring is a very nice example where the electronic motion is confined and the transport becomes predominantly coherent. Using two such metallic rings, we can design a double quantum ring, and, here we will show how such a simple geometric model can be used to design an AND logic gate. To explore this phenomenon, we construct a bridge system where the double quantum ring is sandwiched between two external electrodes (Fig. 1). The theoretical description of electron transfer in a bridge system has got much progress following the pioneering work of Aviram and Ratner [1. Later, several excellent experiments [2, 3, 4] have been done in several bridge systems to understand the basic mechanisms underlying the electron transport. Though in literature many theoretical [5, 6, 7, 8, 9, 10, 11, 12, 13, 14, 15, 16, 17, 18, 19. as well as experimental papers [2, 3, 4, on electron transport are available, yet lot of controversies are still present between the theory and experiment, and the complete knowledge of the conduction mechanism in this scale is not very well established even today.

The aim of the present work is to describe the AND gate response in a double quantum ring where each ring is threaded by a magnetic flux $\phi$. The rings are contacted symmetrically to the electrodes, and the lower arms of the two rings are subjected to two gate voltages $V_{a}$ and $V_{b}$, respectively (see Fig. (1) those are treated as the two inputs of the AND gate. Here we adopt a simple tight-binding model to describe the system and all the calculations are done numerically based on the Green's function formalism. The AND gate response is illustrated by studying the conductance-energy and current-voltage characteristics in terms of the ringto-electrode coupling strengths, magnetic flux and gate voltages. Our study reveals that for a typical value of the magnetic flux, $\phi=\phi_{0} / 2$, a high output current (1) (in the logical sense) is available only if both the two inputs to the gate are high (1), while if neither or only one input to the gate is high (1), a low output current (0) appears. This phenomenon clearly demonstrates the AND gate response, and it may be utilized in designing an electronic logic gate. To the best of our knowledge the AND gate response in such a simple system has not yet been described in the literature.

The scheme of the present paper is as follow. Following the introduction (Section 1), in Section 2, we describe the model and the theoretical formulations for our calculations. Section 3 presents the significant results, and finally, we conclude our results in Section 4.

\section{Model and the synopsis of the theoretical background}

We start by referring to Fig. 1. A double quantum ring, where each ring is threaded by a magnetic flux $\phi$, is attached symmetrically to two semi-infinite one-dimensional (1D) metallic electrodes, namely, source and drain. Two gate electrodes, viz, gate-a and gate-b, are placed below the lower arms of the two rings, respectively, and they are ideally isolated from the rings. The atomic sites $a$ and $b$ in the lower arms of the two rings are subjected to the gate voltages $V_{a}$ and $V_{b}$ via the gate electrodes gate-a and gate-b, respectively, and they are treated as the two inputs of the AND gate. In the present scheme, we consider that the gate voltages each operate on the atomic sites nearest to the plates only. While, in complicated geometric models, the effect must be taken into account for the other dots, though the effect becomes too small. The actual scheme of connections with the batteries for the operation of the AND gate is clearly presented in the figure (Fig. 11), where the source and the gate voltages are applied with respect to the drain.

We calculate the conductance $(g)$ of the double quantum ring using the Landauer conductance formula 20, 21. At much low temperatures and bias voltage it can be expressed as,

$$
g=\frac{2 e^{2}}{h} T
$$

where $T$ gives the transmission probability of an 
electron across the double quantum ring. In terms of the Green's function of the double quantum ring and its coupling to the electrodes, the transmission probability can be written in the form [20, 21],

$$
T=\operatorname{Tr}\left[\Gamma_{S} G_{R}^{r} \Gamma_{D} G_{R}^{a}\right]
$$

where $G_{R}^{r}$ and $G_{R}^{a}$ are respectively the retarded and advanced Green's functions of the double quantum ring including the effects of the electrodes. Here $\Gamma_{S}$ and $\Gamma_{D}$ describe the coupling of the double quan-

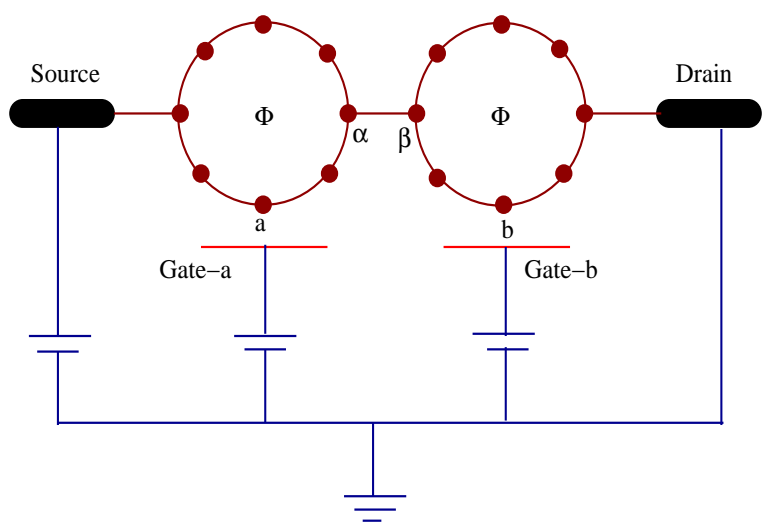

Figure 1: (Color online). The scheme of connections with the batteries for the operation of the AND gate. A double quantum ring is attached to two semi-infinite 1D metallic electrodes, namely, source and drain. The gate voltages $V_{a}$ and $V_{b}$, those are variable, are applied in the atomic sites $a$ and $b$ via the gate electrodes, gate-a and gate-b, respectively. The source and the gate voltages are applied with respect to the drain.

tum ring to the source and drain, respectively. For the full system i.e., the double quantum ring, source and drain, the Green's function is expressed as,

$$
G=(E-H)^{-1}
$$

where $E$ is the injecting energy of the source electron. Evaluation of this Green's function needs the inversion of an infinite matrix since the full system consists of the finite double quantum ring and the two semi-infinite 1D electrodes. However, the full system can be partitioned into sub-matrices corresponding to the individual sub-systems and the Green's function for the double quantum ring can be effectively written in the form,

$$
G_{R}=\left(E-H_{R}-\Sigma_{S}-\Sigma_{D}\right)^{-1}
$$

where $H_{R}$ corresponds to the Hamiltonian of the double quantum ring. Within the non-interacting picture the Hamiltonian can be expressed like,

$$
\begin{aligned}
H_{R} & =\sum_{i}\left(\epsilon_{i}+V_{a} \delta_{i a}+V_{b} \delta_{i b}\right) c_{i}^{\dagger} c_{i} \\
& +\sum_{<i j>} t\left(c_{i}^{\dagger} c_{j} e^{i \theta}+c_{j}^{\dagger} c_{i} e^{-i \theta}\right)
\end{aligned}
$$

In this Hamiltonian $\epsilon_{i}$ 's are the site energies for all the sites $i$ except the sites $i=a$ and $b$ where the gate voltages $V_{a}$ and $V_{b}$ are applied, those are variable. These gate voltages can be incorporated through the site energies as expressed in the above Hamiltonian. $c_{i}^{\dagger}\left(c_{i}\right)$ is the creation (annihilation) operator of an electron at the site $i$ and $t$ is the hopping strength between the nearest-neighbor sites in each ring. The hopping strength between the two atomic sites $(\alpha$ and $\beta$ ) through which the rings are coupled to each other is also set to $t$, for the sake of simplicity. $\theta=2 \pi \phi / N \phi_{0}$ is the phase factor due to the flux $\phi$ in each ring, where $N$ represents the total number of atomic sites (filled circles) in a single ring. A similar kind of tight-binding Hamiltonian is also used, except the phase factor $\theta$, to describe the semi-infinite 1D perfect electrodes where the Hamiltonian is parametrized by constant on-site potential $\epsilon^{\prime}$ and nearest-neighbor hopping integral $t^{\prime}$. The hopping integral between the source and the double quantum ring is $\tau_{S}$, while it is $\tau_{D}$ between the double quantum ring and the drain. The parameters $\Sigma_{S}$ and $\Sigma_{D}$ in Eq. (4) represent the self-energies due to the coupling of the double quantum ring to the source and drain, respectively, where all the information of the coupling are included into these self-energies.

To evaluate the current $(I)$, passing through the double quantum ring, as a function of the applied bias voltage $(V)$ we use the relation [20,

$$
I(V)=\frac{e}{\pi \hbar} \int_{E_{F}-e V / 2}^{E_{F}+e V / 2} T(E, V) d E
$$

where $E_{F}$ is the equilibrium Fermi energy. Here we make a realistic assumption that the entire voltage is dropped across the ring-electrode interfaces, and it is examined that under such an assumption the $I-V$ characteristics do not change their qualitative features.

In this presentation, all the results are computed only at absolute zero temperature. These results are also valid even for some finite (low) temperatures, since the broadening of the energy levels of the double quantum ring due to its coupling to the electrodes becomes much larger than that of 
the thermal broadening 20. On the other hand, at high temperature limit, all these features completely disappear. This is due to the fact that the phase coherence length decreases significantly with the rise of temperature where the contribution comes mainly from the scattering on phonons, and therefore, the quantum interference effect vanishes. For the sake of simplicity, we take the unit $c=e=h=1$ in our present calculations.

\section{Results and discussion}

In order to illustrate the results, let us begin our discussion by mentioning the values of the different parameters used for the numerical calculations. In the double quantum ring, the on-site energy $\epsilon_{i}$ is fixed to 0 for all the sites $i$, except the sites $i=a$ and $b$ where the site energies are taken as $V_{a}$ and $V_{b}$, respectively, and the nearest-neighbor hopping strength $t$ is set to 3 . While, for the side-attached
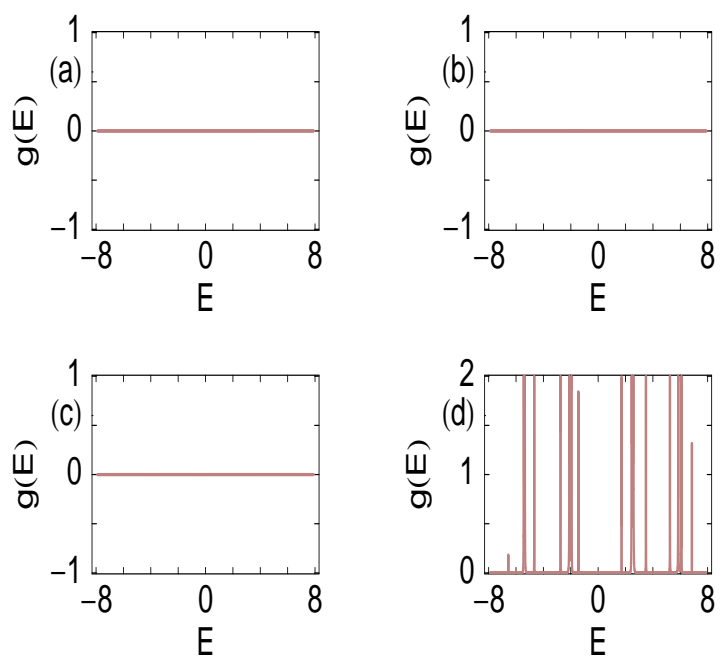

Figure 2: (Color online). Conductance $g$ as a function of the energy $E$ for a double quantum ring with $M=16$ and $\phi=0.5$ in the limit of weak-coupling. (a) $V_{a}=V_{b}=0$, (b) $V_{a}=2$ and $V_{b}=0$, (c) $V_{a}=0$ and $V_{b}=2$ and (d) $V_{a}=V_{b}=2$.

electrodes the on-site energy $\left(\epsilon^{\prime}\right)$ and the nearestneighbor hopping strength $\left(t^{\prime}\right)$ are chosen as 0 and 4 , respectively. The Fermi energy $E_{F}$ is taken as 0 . Throughout the study, we narrate our results for the two limiting cases depending on the strength of the coupling of the double quantum ring to the source and drain. Case $I: \tau_{S(D)}<<t$. It is the so-called weak-coupling limit. For this regime we choose $\tau_{S}=$
$\tau_{D}=0.5$. Case $I I: \tau_{S(D)} \sim t$. This is the socalled strong-coupling limit. In this particular limit, we set the values of the parameters as $\tau_{S}=\tau_{D}=$ 2.5. The key controlling parameter for all these calculations is the magnetic flux $\phi$, threaded by each single ring, which is fixed at $\phi_{0} / 2$ i.e., 0.5 in our chosen unit $c=e=h=1$.

As illustrative examples, in Fig. 2 we plot the conductance-energy $(g-E)$ characteristics for a double quantum ring with $M=16(M=2 N$, the total number of atomic sites in the double quantum ring, since each ring contains $N$ atomic sites) in the limit of weak-coupling, where (a), (b), (c) and (d) correspond to the results for the four different
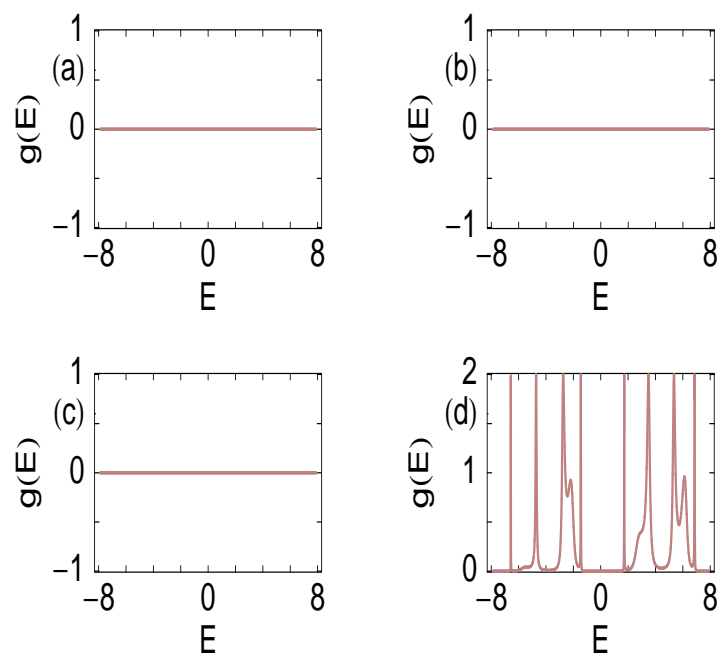

Figure 3: (Color online). Conductance $g$ as a function of the energy $E$ for a double quantum ring with $M=16$ and $\phi=0.5$ in the limit of strong-coupling. (a) $V_{a}=V_{b}=0$, (b) $V_{a}=2$ and $V_{b}=0$, (c) $V_{a}=0$ and $V_{b}=2$ and (d) $V_{a}=V_{b}=2$.

choices of the gate voltages $V_{a}$ and $V_{b}$, respectively. When both the two inputs $V_{a}$ and $V_{b}$ are identical to zero i.e., both the inputs are low, the conductance $g$ becomes exactly zero for the entire energy range (see Fig. 2(a)). A similar response is also observed for the other two cases where anyone of the two inputs $\left(V_{a}\right.$ and $\left.V_{b}\right)$ to the gate is high and other one is low. The results are shown in Figs. 2(b) and (c), respectively. Thus for all these three cases (Figs. 2(a)(c)), the double quantum ring does not allow to pass an electron from the source to the drain. The conduction of electron through the bridge system is allowed only when both the two inputs to the gate are high i.e., $V_{a}=V_{b}=2$. The response is given in Fig. 2(d), and it is observed that for some particu- 
lar energies the conductance exhibits fine resonant peaks. At the resonant energies, the conductance approaches the value 2 , and accordingly, the transmission probability $T$ goes to unity, since the relation $g=2 T$ holds from the Landauer conductance formula (see Eq. (1) with $e=h=1$ ). All these resonant peaks are associated with the energy eigenvalues of the double quantum ring, and therefore, we can predict that the conductance spectrum manifests itself the electronic structure of the double
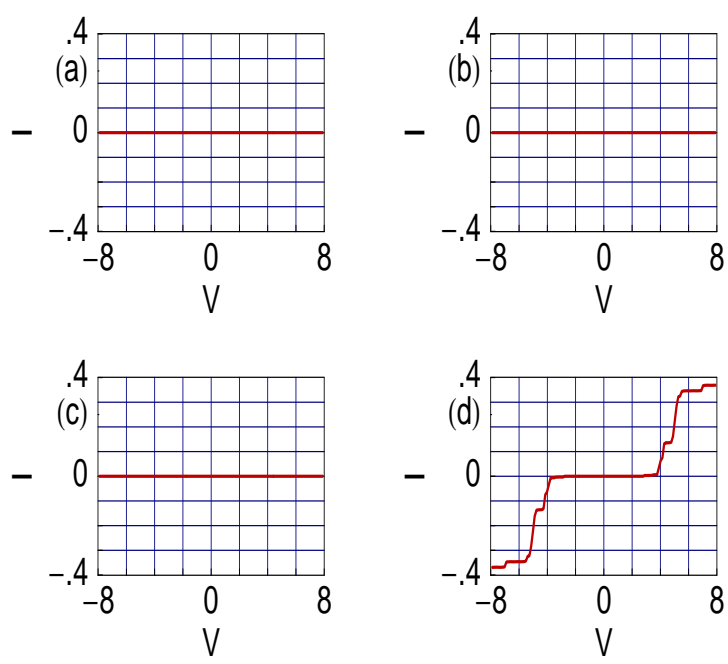

Figure 4: (Color online). $I-V$ characteristics for a double quantum ring with $M=16$ and $\phi=0.5$ in the weak-coupling limit. (a) $V_{a}=V_{b}=0$, (b) $V_{a}=2$ and $V_{b}=0$, (c) $V_{a}=0$ and $V_{b}=2$ and (d) $V_{a}=V_{b}=2$.

quantum ring. Now we try to figure out the dependences of the gate voltages on the electron transport in these four different cases. The probability amplitude of getting an electron from the source to drain across the double quantum ring depends on the combined effect of the quantum interferences of the electronic waves passing through the upper and lower arms of the two rings. For a symmetrically connected ring (length of the two arms of the ring are identical to each other) which is threaded by a magnetic flux $\phi$, the probability amplitude of getting an electron across the ring becomes exactly zero $(T=0)$ for the typical flux, $\phi=\phi_{0} / 2$. This is due to the result of the quantum interference among the two waves in the two arms of the ring, which can be shown through few simple mathematical steps. Thus for the particular case when both the two inputs to the gate are low (0), the upper and lower arms of the two rings become exactly identical, and accordingly, the transmission probability vanishes. The similar response i.e., the vanishing transmission probability, is also achieved for the two other cases $\left(V_{a}=2, V_{b}=0\right.$ and $\left.V_{a}=0, V_{b}=2\right)$, where the symmetry is broken only in one ring out of these two by applying a gate voltage either in the site $a$ or in $b$, preserving the symmetry in the other ring. The reason is that, when anyone of the two gates $\left(V_{a}\right.$ and $V_{b}$ ) is non-zero, the symmetry between the upper and lower arms is broken only in one ring which provides non-zero transmission probability across the ring. While, for the other ring where no gate voltage is applied, the symmetry between the two arms becomes preserved which gives zero transmission probability. Accordingly, the combined effect provides vanishing transmission probability across

Table 1: AND gate response in the weak-coupling limit. The current $I$ is computed at the bias voltage 6.02 .

\begin{tabular}{|c|c|c|}
\hline \hline Input-I $\left(V_{a}\right)$ & Input-II $\left(V_{b}\right)$ & Current $(I)$ \\
\hline 0 & 0 & 0 \\
\hline 2 & 0 & 0 \\
\hline 0 & 2 & 0 \\
\hline 2 & 2 & 0.346 \\
\hline \hline
\end{tabular}

the bridge, as the two rings are coupled to each other. The non-zero value of the transmission probability is achieved only when the symmetries of both the two rings are identically broken. This can be done by applying the gate voltages in both the sites $a$ and $b$ of the two rings. Thus for the particular case when both the two inputs are high i.e., $V_{a}=V_{b}=2$, the non-zero value of the transmission probability appears. This feature clearly demonstrates the AND gate behavior. With these characteristics, we get additional one feature when the coupling strength of the double quantum ring to the electrodes increases from the low regime to the high one. In the limit of strong-coupling, all these resonant peaks get substantial widths compared to the weak-coupling limit. The results are shown in Fig. 3. where all the other parameters are identical to those in Fig. 22 The contribution for the broadening of the resonant peaks in this strong-coupling limit appears from the imaginary parts of the selfenergies $\Sigma_{S}$ and $\Sigma_{D}$, respectively [20. Hence by tuning the coupling strength, we can get the electron transmission across the double quantum ring for the wider range of energies and it provides an 
important signature in the study of current-voltage $(I-V)$ characteristics.

All these features of electron transfer become much more clearly visible by studying the $I-V$ characteristics. The current passing through the double quantum ring is computed from the integration procedure of the transmission function $T$ as prescribed in Eq. (6). The transmission function varies exactly similar to that of the conductance spectrum, differ only in magnitude by the factor 2 since the relation $g=2 T$ holds from the Landauer conductance formula Eq. (11). As representative examples, in Fig. 4 we plot the current $I$ as a function of the applied bias voltage $V$ for a double quantum ring considering $M=16$ in the limit of weak-coupling, where (a), (b), (c) and (d) represent the results for the four different cases of the two gate volt-
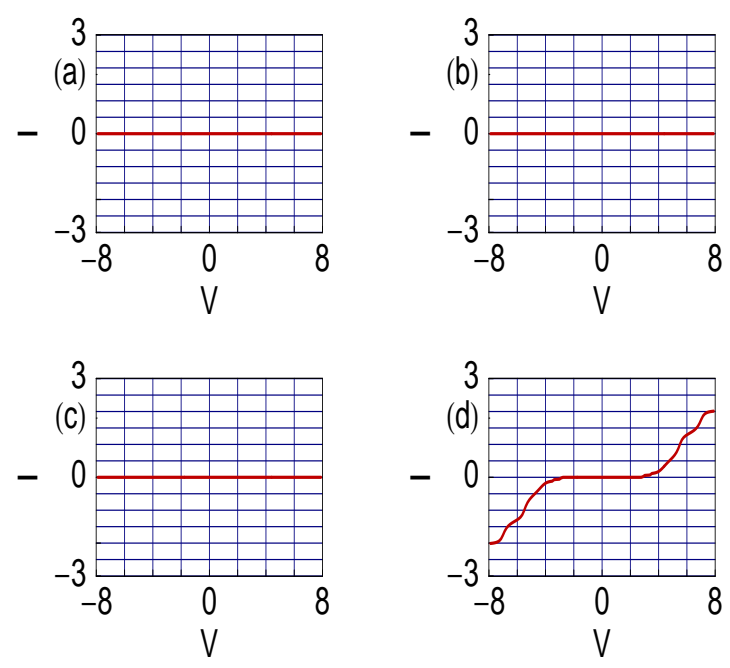

Figure 5: (Color online). $I-V$ characteristics for a double quantum ring with $M=16$ and $\phi=0.5$ in the strong-coupling limit. (a) $V_{a}=V_{b}=0$, (b) $V_{a}=2$ and $V_{b}=0$, (c) $V_{a}=0$ and $V_{b}=2$ and (d) $V_{a}=V_{b}=2$.

ages $V_{a}$ and $V_{b}$. For the cases when either both the two inputs to the gate are low $\left(V_{a}=V_{b}=0\right)$, or anyone of the two inputs is high and other is low $\left(V_{a}=2, V_{b}=0\right.$ or $\left.V_{a}=0, V_{b}=2\right)$, the current is exactly zero for the entire range of the bias voltage. The results are shown in Figs. 4(a)-(c), and, the vanishing behavior of the current in these three cases can be clearly understood from the conductance spectra Figs. 2(a)-(c), since the current is computed from the integration procedure of the transmission function $T$. The non-vanishing current amplitude is observed only for the typical case where both the two inputs to the gate are high i.e., $V_{a}=V_{b}=2$. The result is shown in Fig. 4(d). From this figure it is observed that the current exhibits staircase-like structure with fine steps as a function of the applied bias voltage. This is due to the existence of the sharp resonant peaks in the conductance spectrum in the weak-coupling limit, since the current is computed by the integration method of the transmission function $T$. With the increase of the bias voltage $V$, the electrochemical potentials on the electrodes are shifted gradually, and finally cross one of the quantized energy levels of the double quantum ring. Therefore, a current channel is

Table 2: AND gate response in the strong-coupling limit. The current $I$ is computed at the bias voltage 6.02 .

\begin{tabular}{|c|c|c|}
\hline \hline Input-I $\left(V_{a}\right)$ & Input-II $\left(V_{b}\right)$ & Current $(I)$ \\
\hline 0 & 0 & 0 \\
\hline 2 & 0 & 0 \\
\hline 0 & 2 & 0 \\
\hline 2 & 2 & 1.295 \\
\hline \hline
\end{tabular}

opened up which provides a jump in the $I$ - $V$ characteristic curve. Addition to these behaviors, it is also important to note that the non-zero value of the current appears beyond a finite value of $V$, the so-called threshold voltage $\left(V_{t h}\right)$. This $V_{t h}$ can be controlled by tuning the size $(N)$ of the two rings. From these $I-V$ characteristics, the behavior of the AND gate response is clearly visible. To make it much clear, in Table1, we present a quantitative estimate of the typical current amplitude, computed at the bias voltage $V=6.02$, in this weak-coupling limit. It shows $I=0.346$ only when both the two inputs to the gate are high $\left(V_{a}=V_{b}=2\right)$, while for the other three cases when either $V_{a}=V_{b}=0$ or $V_{a}=2, V_{b}=0$ or $V_{a}=0, V_{b}=2$, the current $I$ gets the value 0 . In the same footing, as above, here we also discuss the $I-V$ characteristics in the limit of strong-coupling. In this limit, the current varies almost continuously with the applied bias voltage and achieves much larger amplitude than the weakcoupling case (Fig. 4) as presented in Fig. 5, The reason is that, in the limit of strong-coupling all the resonant peaks get broadened which provide larger current in the integration procedure of the transmission function $T$. Thus by tuning the strength of the ring-to-electrodes coupling, we can achieve very large current amplitude from the very low one 
for the same bias voltage $V$. All the other properties i.e., the dependences of the gate voltages on the $I-V$ characteristics are exactly similar to those as given in Fig. 4. In this strong-coupling limit we also make a quantitative study for the typical current amplitude, given in Table 2] where the current amplitude is determined at the same bias voltage $(V=6.02)$ as earlier. The response of the output current is exactly similar to that as given in Table 1. Here the non-zero value of the current gets the value 1.295 which is much larger compared to the weakcoupling case which shows the value 0.346. From these results we can clearly manifest that a double quantum ring exhibits the AND gate response.

\section{Concluding remarks}

To summarize, we have addressed the AND gate behavior in a double quantum ring where each ring is threaded by a magnetic flux $\phi$. The double quantum ring is attached symmetrically to two semiinfinite 1D metallic electrodes and two gate voltages, namely, $V_{a}$ and $V_{b}$, are applied, respectively, in the lower arms of the two rings and they are considered as the two inputs of the AND gate. The full system is described by the tight-binding model and all the calculations are done in the Green's function formalism. We have numerically computed the conductance-energy and current-voltage characteristics as functions of the ring-electrode coupling strengths, magnetic flux and gate voltages. Very interestingly we have noticed that, for the half fluxquantum value of $\phi\left(\phi=\phi_{0} / 2\right)$, a high output current (1) (in the logical sense) appears only if both the inputs to the gate are high (1). On the other hand, if neither or only one input to the gate is high (1), a low output current (0) results. It clearly manifests the AND gate response, and, this aspect may be utilized in designing a tailor made electronic logic gate. In view of the potential application of this AND gate as a circuit element in an integrated circuit, we would like to mention that care should be taken during the application of the magnetic field in the two rings such that the other circuit elements of the integrated circuit are not affected by this field.

Throughout our work, we have addressed the conductance-energy and current-voltage characteristics for a double quantum ring with total number of atomic sites $M=16$. In our model calculations, this typical number $(M=16)$ is chosen only for the sake of simplicity. Though the results presented here change numerically with the ring size $(N)$, but all the basic features remain exactly invariant. To be more specific, it is important to note that, in real situation the experimentally achievable rings have typical diameters within the range 0.4-0.6 $\mu \mathrm{m}$. In such a small ring, unrealistically very high magnetic fields are required to produce a quantum flux. To overcome this situation, Hod et al. have studied extensively and proposed how to construct nanometer scale devices, based on Aharonov-Bohm interferometry, those can be operated in moderate magnetic fields [22, 23, 24, 25].

In the present paper we have done all the calculations by ignoring the effects of the temperature, electron-electron correlation, disorder, etc. Due to these factors, any scattering process that appears in the arms of the rings would have influence on electronic phases, and, in consequences can disturb the quantum interference effects. Here we have assumed that, in our sample all these effects are too small, and accordingly, we have neglected all these factors in this particular study.

The importance of this article is mainly concerned with (i) the simplicity of the geometry and (ii) the smallness of the size. To the best of our knowledge the AND gate response in such a simple low-dimensional system that can be operated even at finite temperatures (low) has not been addressed earlier in the literature.

\section{References}

[1] A. Aviram and M. Ratner, Chem. Phys. Lett. 29, 277 (1974).

[2] T. Dadosh, Y. Gordin, R. Krahne, I. Khivrich, D. Mahalu, V. Frydman, J. Sperling, A. Yacoby and I. Bar-Joseph, Nature 436, 677 (2005).

[3] J. Chen, M. A. Reed, A. M. Rawlett and J. M. Tour, Science 286, 1550 (1999).

[4] M. A. Reed, C. Zhou, C. J. Muller, T. P. Burgin and J. M. Tour, Science 278, 252 (1997).

[5] P. A. Orellana, M. L. Ladron de Guevara, M. Pacheco and A. Latge, Phys. Rev. B 68, 195321 (2003).

[6] P. A. Orellana, F. Dominguez-Adame, I. Gomez and M. L. Ladron de Guevara, Phys. Rev. B 67, 085321 (2003).

[7] A. Nitzan, Annu. Rev. Phys. Chem. 52, 681 (2001). 
[8] A. Nitzan and M. A. Ratner, Science 300, 1384 (2003).

[9] D. M. Newns, Phys. Rev. 178, 1123 (1969).

[10] V. Mujica, M. Kemp and M. A. Ratner, J. Chem. Phys. 101, 6849 (1994).

[11] V. Mujica, M. Kemp, A. E. Roitberg and M. A. Ratner, J. Chem. Phys. 104, 7296 (1996).

[12] K. Walczak, Phys. Stat. Sol. (b) 241, 2555 (2004).

[13] K. Walczak, arXiv:0309666.

[14] W. Y. Cui, S. Z. Wu, G. Jin, X. Zhao and Y. Q. Ma, Eur. Phys. J. B. 59, 47 (2007).

[15] R. Baer and D. Neuhauser, J. Am. Chem. Soc. 124, 4200 (2002).

[16] D. Walter, D. Neuhauser and R. Baer, Chem. Phys. 299, 139 (2004).

[17] K. Tagami, L. Wang and M. Tsukada, Nano Lett. 4, 209 (2004).

[18] K. Walczak, Cent. Eur. J. Chem. 2, 524 (2004).

[19] R. Baer and D. Neuhauser, Chem. Phys. 281, 353 (2002).

[20] S. Datta, Electronic transport in mesoscopic systems, Cambridge University Press, Cambridge (1997).

[21] M. B. Nardelli, Phys. Rev. B 60, 7828 (1999).

[22] O. Hod, R. Baer and E. Rabani, J. Phys. Chem. B 108, 14807 (2004).

[23] O. Hod, R. Baer and E. Rabani, J. Phys.: Condens. Matter 20, 383201 (2008).

[24] O. Hod, R. Baer and E. Rabani, J. Am. Chem. Soc. 127, 1648 (2005).

[25] O. Hod, E. Rabani and R. Baer, Acc. Chem. Res. 39, 109 (2006). 